\title{
Theoretical and Experimetal Hints to a Flux of Doppler-Transformation-Energies during processes with Energy Conservation
}

Christoph Schultheiss ( $\square$ eucschult@yahoo.de )

Karlsruhe Institute of Technoloy (KIT)

Article

Keywords: Compton-Effect, Doppler-Effect, energy- momentum conservation, flywheel

Posted Date: November 13th, 2020

DOl: https://doi.org/10.21203/rs.3.rs-105656/v1

License: (1) (1) This work is licensed under a Creative Commons Attribution 4.0 International License.

Read Full License 
Theoretical and Experimental Hints to a Flux of Doppler-Transformation-Energies during Processes with Energy Conservation

\title{
Christoph Schultheiss
}

\author{
Karlsruhe Institute of Technology (KIT). Institute for Pulsed Power and Microwave \\ Technology (IHM), P.O. Box 3640, 76021 Karlsruhe, Germany
}

\begin{abstract}
In a microscopic model of the photoelectric effect it becomes clear that the conservation of energy is exclusively determined by Doppler shift processes, i.e., the whole energy of the photon vanishes by means of Doppler redshifts. Accordingly, if a photon is generated, the energy is won by Doppler blueshifts. This is supposed to be valid for all processes with energy conservation. An experiment is carried out to make this Doppler energy flow visible by means of interactions with probes. The result of this experiment is that a weak force is measurable in the vicinity of processes with energy conservation. With the aid of a twisted rubber driven low-power device $(\tilde{\mathrm{P}}=10 \mathrm{~W})$, periodic accelerations and decelerations of about $10^{-6}$ $\mathrm{m} / \mathrm{s}^{2}$ are measurable. In the close vicinity of the device, accelerations with values up to $10^{-3}$ $\mathrm{m} / \mathrm{s}^{2}$ can be concluded. The consequences which result from this force are discussed.
\end{abstract}

Keywords: Compton-Effect, Doppler-Effect, energy- momentum conservation, flywheel

\section{Introduction}

The Doppler Effect is investigated very well and is widely applied in science and technology. An outstanding example of its applications is the Mößbauer Effect [1], where velocities down to $1 \mathrm{~mm} / \mathrm{s}$ are easily measurable.

Schrödinger [2] as well as other authors analyzed in the early $20^{\text {th }}$ century that during a Compton collision between a charged particle and a photon the change of photon energy relates exactly to a Doppler shift $[3,4,5,6,7]$. The shift corresponds to a direct increase or decrease of the wavelength in a red- or blueshift situation, respectively. However, the role of the Doppler shift in physics seems to go deeper as it appears at first glance. In this paper we try to gather new aspects of this kind of relativistic transformation.

2. Short derivation of the Compton shift as a Doppler shift

In Fig.1, let $\mathrm{M}$ be a big and $\mathrm{m}$ a small mass - both shall be charged particles. A photon reflects between both masses comprised with ideally reflecting mirrors. In addition, the

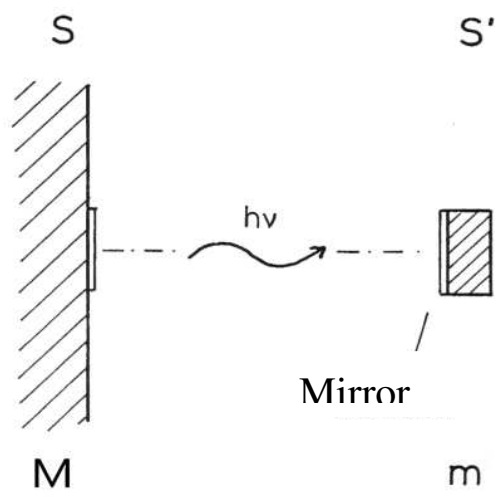

Fig.l Arrangement of mirrors, masses and photon in the Mirror Mass Model 
mirrors are mutually aligned, so that the photon cannot escape during the reflections (see Fig.1). As a consequence of multiple reflections, the small mass $m$ starts to accelerate away from the big mass $M$ and the kinetic energy successively approaches $\frac{1}{2} \mathrm{mv}^{2} \rightarrow \mathrm{h} v[8,9]$ provided the lateral dimension of the mirrors are unlimited. This is a model of the photoelectric effect proposed to be called Mirror Mass Model (MMM).

After the first collision with the photon the small mass m moves with the velocity $v_{1}=\beta_{1} \cdot c$ and the interacting photon, coming back from the moving charge, becomes Compton shifted or Doppler redshifted corresponding to $\beta_{1}$. This is demonstrated for the special case of an elastic $180^{\circ}$ Compton collision:

With the abbreviations $\alpha_{0}=\frac{\mathrm{h} v_{\mathrm{o}}}{\mathrm{mc}^{2}}, \gamma_{1}=\left(1-\beta_{1}^{2}\right)^{-1 / 2}$ and for a system initially at rest $\left(\beta_{0}=0\right)$, the energy and momentum equations have the form Eq.1and 2. By mutual addition and subtraction of both energy and momentum conservation equations

$$
\begin{aligned}
& \alpha_{0}+1=\alpha_{1}+\gamma_{1} \\
& \alpha_{0}+0=-\alpha_{1}+\beta_{1} \gamma_{1}
\end{aligned}
$$

and using the defining equation $\gamma_{1}+\beta_{1} \gamma_{1}=\left(\gamma_{1}-\beta_{1} \gamma_{1}\right)^{-1}$ this leads to the well-known solutions:

$$
\begin{aligned}
& \alpha_{1}=\alpha_{0}\left(1+2 \alpha_{0}\right)^{-1} \\
& \gamma_{1}=1+2 \alpha_{0}^{2}\left(1+2 \alpha_{0}\right)^{-1}
\end{aligned}
$$

To make the Doppler mechanism visible, Eq. 1 and 2 can be rewritten as

$$
\begin{aligned}
& \gamma_{1}=\alpha_{0}-\alpha_{1}+1 \\
& \beta_{1} \gamma_{1}=\alpha_{0}+\alpha_{1} .
\end{aligned}
$$

Subtraction and addition lead to Doppler transformation-like expressions:

$$
\begin{aligned}
& \gamma_{1}-\beta_{1} \gamma_{1}=1-2 \alpha_{1} \\
& \gamma_{1}+\beta_{1} \gamma_{1}=1+2 \alpha_{0} .
\end{aligned}
$$

Using again the defining equation $\gamma_{1}+\beta_{1} \gamma_{1}=\left(\gamma_{1}-\beta_{1} \gamma_{1}\right)^{-1}$, it follows that

$$
\gamma_{1}-\beta_{1} \gamma_{1}=1-2 \alpha_{1}=\left(1+2 \alpha_{0}\right)^{-1} \text {. }
$$

Inserting this into Eq.7 and comparing to Eq.3, this gives:

$$
\gamma_{1}-\beta_{1} \gamma_{1}=\frac{\alpha_{1}}{\alpha_{0}} \equiv D_{1}
$$

which is the Doppler equation with symbol D. Compton shift is a Doppler shift. But the role of the Doppler Effect is bigger still: The hypothesis of reference [8] demands: Any change of photon energy is exclusively caused by Doppler transformations processes.

\section{The DCAD-Method}

Supported by the ladder statement, the Compton process is proposed to be expanded into a number of single Doppler processes. The method to make this visible will be called DCAD. It stands for:

Doppler transformation of the photon from system $S$ into the moved system $S^{\prime}\left(D_{S^{\prime}}\right)$ Compton collision in the system $S^{\prime}$; the system gains the Compton velocity ( $\tilde{D}$ ) Addition (relativistic) of Compton- and Doppler velocity gives the backtransformation velocity $\left(\tilde{D} \cdot D_{S^{\prime} S}\right)$ 
Doppler back-transformation of the photon from the system $S^{\prime}$ to the system $S$

As seen from the system $S$ in the arrangement of Fig. 1, the sequence $\alpha_{0}, \alpha_{1}, \alpha_{2}, \alpha_{3} .$. and so on can be expressed in products of Doppler transformations. For example, the first turn has the form:

$$
\alpha_{1}=\mathrm{D}_{\mathrm{SS}^{\prime}} \cdot \tilde{\mathrm{D}}_{1} \cdot \mathrm{D}_{\mathrm{S}^{\prime} \mathrm{S}} \cdot \alpha_{0} \quad \mathrm{D}_{1} \leq 1
$$

Here, $D_{\mathrm{SS}^{\prime}}$ is the transformation of the photon from the system $\mathrm{S}$ into the system $\mathrm{S}^{\prime}$ The term $\tilde{D}_{1}$ is the Doppler shift coming from the Compton velocity, seen in the system $\mathrm{S}^{\prime}$. The velocity addition $\tilde{D}_{1} \cdot D_{S^{\prime} S}$ stands for the back-transformation. Doppler shifts with the index $S^{\prime} S$ and $S^{\prime} S^{\prime}$ are equal in value and can in principle be written in Eq.11 as $D_{0}^{2}$, which also means that the photon suffered two Doppler red-shifts - on the way from $S$ to $S^{\prime}$ and the other one on the way back.

The Compton- or Doppler shift $\tilde{D}_{1}$ itself is defined in Eq.10. The property $\gamma_{1}$ is determined by Eq.4 and $\beta_{1}$ by means of the definition equation $\beta_{1}\left(\gamma_{1}\right)=\left(1-\gamma_{1}^{-2}\right)^{\frac{1}{2}}$, which gives finally:

$$
\tilde{\mathrm{D}}_{1}=\frac{1}{1+2 \alpha_{0}}
$$

where $\alpha_{0}$ is the photon energy in the frame S'. In the vein of Eq.11 the first three collisions have the form:

$$
\begin{array}{lc}
\alpha_{1}=\mathrm{D}_{0} \tilde{\mathrm{D}}_{1} \mathrm{D}_{0} \cdot \alpha_{0}=\tilde{\mathrm{D}}_{1} \mathrm{D}_{0}^{2} \cdot \alpha_{0} & \tilde{\mathrm{D}}_{1}=\frac{1}{1+2 \alpha_{0}} \\
\alpha_{2}=\mathrm{D}_{1} \tilde{\mathrm{D}}_{2} \mathrm{D}_{1} \cdot \alpha_{1}=\mathrm{D}_{0} \tilde{\mathrm{D}}_{1} \cdot \tilde{\mathrm{D}}_{2} \cdot \mathrm{D}_{0} \tilde{\mathrm{D}}_{1} \cdot \underbrace{\mathrm{D}_{0} \tilde{\mathrm{D}}_{1} \mathrm{D}_{0}}_{\mathrm{D}_{2}} \cdot \alpha_{0}=\tilde{\mathrm{D}}_{2} \tilde{\mathrm{D}}_{1}^{3} \mathrm{D}_{0}^{4} \cdot \alpha_{0} & \tilde{\mathrm{D}}_{2}=\frac{1}{1+2 \alpha_{0} \mathrm{D}_{0}^{2} \tilde{\mathrm{D}}_{1}} \\
\begin{array}{c}
\mathrm{D}_{3}=\mathrm{D}_{2} \tilde{\mathrm{D}}_{3} \mathrm{D}_{2} \cdot \alpha_{2}=\underbrace{\mathrm{D}_{0} \tilde{\mathrm{D}}_{1} \tilde{\mathrm{D}}_{2}}_{\mathrm{D}_{3}} \cdot \tilde{\mathrm{D}}_{3} \cdot \underbrace{\mathrm{D}_{0} \tilde{\mathrm{D}}_{1} \tilde{\mathrm{D}}_{2}}_{\mathrm{D}_{2}} \cdot \underbrace{\tilde{\mathrm{D}}_{2} \tilde{\mathrm{D}}_{1}^{3} \mathrm{D}_{0}^{4} \cdot \alpha_{0}}_{\alpha_{2}}=\tilde{\mathrm{D}}_{3} \tilde{\mathrm{D}}_{2}^{3} \tilde{\mathrm{D}}_{1}^{5} \mathrm{D}_{0}^{6} \cdot \alpha_{0} \\
\tilde{\mathrm{D}}_{3}=\frac{1}{1+2 \alpha_{0} \mathrm{D}_{0}^{4} \tilde{\mathrm{D}}_{1}^{3} \tilde{\mathrm{D}}_{2}}
\end{array}
\end{array}
$$

and so on.

In Eq.13, the photon undergoes a series of energy changes in the fashion of a photoelectric effect process. This is a consequence of succeeding Doppler transformations of the form $\mathrm{D}_{0}^{2 \mathrm{n}} \cdot \tilde{\mathrm{D}}_{1}^{2 \mathrm{n}-3} \cdots \tilde{\mathrm{D}}_{\mathrm{n}-2}^{3} \cdot \tilde{\mathrm{D}}_{\mathrm{n}-1}^{2}$. For instance, the exponential growth $\mathrm{D}_{0}^{2 \mathrm{n}}$ indicates that initial Doppler shifts are repeated two times with each turn and so on.

Normally, Doppler transformations are considered to be a very intrinsic behavior of spacetime. But in the photoelectric effect the Doppler mechanism does not appear explicitly in the energy- and momentum conservation equations (see Eqs.1-4), although the upper derivations clearly show the dominant role of Doppler transformations.

In Fig.2 the viewpoint of classical energy conservation versus the presented Doppler model of the photoelectric effect is sketched. 

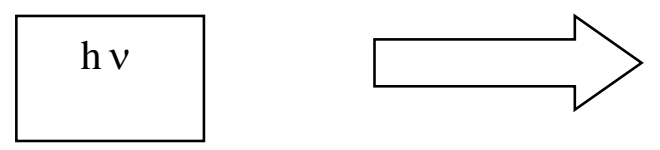

$$
\frac{1}{2} \mathrm{~m} \mathrm{v}^{2}
$$
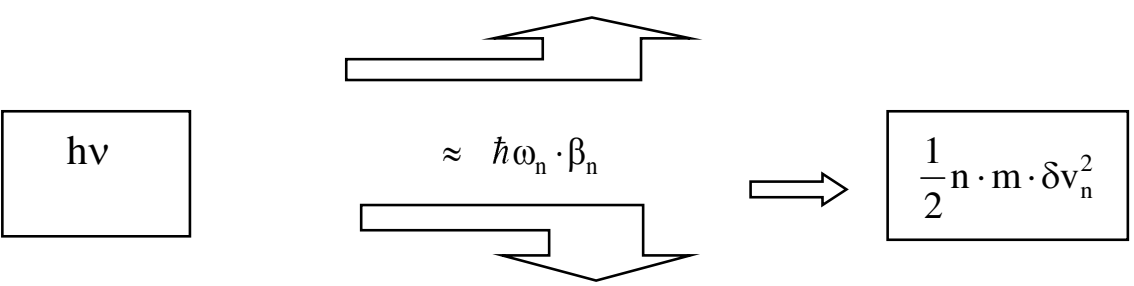

Fig.2: Classical scheme of energy transfer of a photon to a mass $m$ during a photoelectric effect (upper graph), while in the microscopic model the flow of energy into the space is dominant in comparison with the direct step-wise flow on $\mathrm{m}$. This is true especially in nonrelativistic processes (see Eq.14). The mass $m$ itself gains a velocity push $\left(2 \delta v_{n}\right)$ with each collision and approaches successively the final velocity $\mathrm{v}$ after $\mathrm{n}$ reflections.

In the first case by computation all energy of the photon is transferred directly from the photon to the mass $\mathrm{m}$ in form of kinetic energy. It is indispensable from momentum reasons that in a photoelectric effect a heavy mass nearby must be involved to absorb momentum. In the second case an extra heavy mass is not necessary since the big mass $M$ serves to reflect (absorb) the photon momentum without gaining kinetic energy. The velocity of the small mass $\mathrm{m}$ changes step-wise to higher values. An observer in the system $S^{\prime}$ state that the photon coming from the system $S$ has lost energy by Compton red shift. The step wise increase of velocity of the mass amounts in a non-relativistic case to $\delta \mathrm{v}=2 \mathrm{~h} / \mathrm{m} \lambda$. Especially in the non-relativistic case most of the photon energy "is absorbed" in the space by means of Doppler shifts. The quotient $Q$ between the step-wise increase of kinetic energy and the loss due to red shift shows:

$$
\mathrm{Q}=\frac{\frac{1}{2} \cdot \mathrm{m} \cdot \delta \mathrm{v}_{\mathrm{n}}^{2}}{\mathrm{~h} v\left(1-\frac{\mathrm{v}_{\mathrm{n}}}{\mathrm{c}}\right)-\mathrm{h} v}=\frac{\frac{1}{2} \cdot \mathrm{m} \cdot \delta \mathrm{v}_{\mathrm{n}}^{2}}{\mathrm{~h} v \cdot \frac{\mathrm{v}_{\mathrm{n}}}{\mathrm{c}}}=\frac{\frac{1}{2} \cdot \mathrm{m} \cdot \delta \mathrm{v}_{\mathrm{n}}}{\mathrm{m} \cdot \mathrm{v}_{\mathrm{n}}}=\frac{1}{2} \cdot \frac{\delta \mathrm{v}_{\mathrm{n}}}{\mathrm{v}_{\mathrm{n}}}=\frac{1}{2} \frac{\frac{\mathrm{h}}{\mathrm{m} \cdot \lambda_{\mathrm{n}}}}{\sqrt{\frac{1}{2} \cdot \frac{\mathrm{hc}}{\mathrm{m} \lambda_{\mathrm{n}}}}}=\sqrt{\frac{\delta \mathrm{v}_{\mathrm{n}}}{2 \mathrm{c}}} \rightarrow 0
$$

The smaller $\delta \mathrm{v}_{\mathrm{n}}$ the bigger is the fraction of photon energy which is transferred into Doppler red shift energy. This is maximal in low energy processes. The conclusion finally is: Each of these Doppler shift losses can be considered as the emission of something like transformation quanta (see Fig.2).

If we agree the emission of a hypothetic Doppler Transformation Quantum (DTQ) into space, then it will take place during each photon reflection turn ( $S$ to $S^{\prime}$ and vice versa). The Taylor derivation in terms of $\beta$ of Eq.10 gives:

$$
h v^{\prime}=h v \cdot D_{i}=h v \cdot \gamma^{\prime}\left(1-\beta_{i}^{\prime}\right)=h v \frac{1-\beta_{i}^{\prime}}{\sqrt{1-\beta_{i}^{\prime 2}}}=h v\left(1-\beta_{i}^{\prime}\right) \cdot\left(1+\frac{1}{2} \beta_{i}^{\prime 2}-\frac{1}{8} \beta_{i}^{\prime 4}+. .\right)=h v-h v \beta_{i}^{\prime}+\cdots
$$

The thesis is that $h v$ decays in: 


$$
\mathrm{h} v \rightarrow \mathrm{h} v^{\prime}+\underbrace{\mathrm{h} v \cdot \beta_{\mathrm{i}}^{\prime}}_{\text {DTQ }}+\cdots
$$

where the relativistic term $h v \cdot \beta_{i}^{\prime}$ describes the DTQ mentioned above. Obviously in this formalism each photon carries the information about its emission- and collision system. This is because the Doppler term in Eq.16 included already the velocity difference $\beta$ ' between both systems. The question is, when does a Doppler shift happen: at the moment of the emission of the photon, in between or at the moment of collision?

i) Let the Doppler shift take place in the course of the emission, then the collision partner of the photon is already determined in advance, although the collision will take place anywhere (far) in the future. Irregular movements of the collision systems $S^{`}$ during flight time will complicate the situation.

ii) A Doppler shift anywhere between emission and collision increases the problem still more. It needs information about the collision partner and the emission system, too.

iii) The Doppler shift at the moment of collision in the system $S^{`}$ needs the knowledge of the emission system $\mathrm{S}$.

An interesting aspect comes from an investigation of the Compton Effect with a virtual mass that is built from a confined photon in a mirror cavity [9]. It turns out that the re-emission of the Compton photon takes place already in form of a Doppler shifted recoil photon, although the laboratory system has not been entered yet. This means that the photon already knows how the transformation will look like. In this sense, following Eq.16, after the emission the photon has already decayed into a recoil photon and a DTQ. Both energy forms travel side by side until they become separated in the pre-determined reflection of the photon. The DTQ now on its own - continues the propagation in space and disappears finally in the DTQ pool.

The hypothesis is that the space absorbs and emits DTQs as well. In the close vicinity of a collision process, the flux of DTQs should be measurable, if emitted or absorbed DTQs have a finite cross-section for interaction with masses. Furthermore, any acceleration of (solid state) masses is based on the transfer of acceleration forces via electric binding fields from atom layer to atom layer. The imagination is that binding fields between the atom layers are virtual back- and forward reflecting photons. The hypothesis is that it is generally true that these virtual photons also interact with space as described in Fig.1. With other words: in the vicinity of any accelerated mass a flux of DTQs should appear. This will be investigated and tested in an experiment described in Chap 4.

\section{The torsion balance measure system}

The goal of the experiment is to detect very weak forces which appear periodically repulsive and attractive in the vicinity of an oscillating flywheel (Chap.4a). Some basic assumptions must be discussed before. We assume a DTQ power-density $\mathrm{j}$ which should escape or is absorbed tangentially in the direction of an accelerated or decelerated flywheel. The DTQs penetrate a probe mass nearby which shall consist of nucleons. The expected acceleration a of a nucleon which is penetrated by this flow can be estimated by means of the renowned formula in optics for radiation pressure in case of total absorption of radiation: 


$$
a=\frac{\sigma j}{m_{p} c}
$$

The cross-section amounts to $\sigma=1.4 \cdot 10^{-2} \mathrm{~b}=1,4 \cdot 10^{-30} \mathrm{~m}^{2}$, which corresponds to the area of a proton. For a power density of $10^{4} \mathrm{~W} / \mathrm{m}^{2}$, an acceleration of about $3 \cdot 10^{-8} \mathrm{~m} / \mathrm{s}^{2}$ can be expected.

\subsection{Specification of the torsion balance and measure system}

To measure weak forces, a sensitive torsion balance has been constructed. The torsion pendulum is of light plastic with a radius of $4.5 \mathrm{~cm}$. On the one end of the pendulum a lead-tin alloy is mounted and on the other end a small mirror is fixed with its surface directed normally to the radius (see Fig.3 and Fig.5). The weight of each mass is $0.2 \mathrm{~g}$. The total weight of the torsion balance is $0.5 \mathrm{~g}$. The torsion balance is attached to a $27 \mathrm{~cm}$ long fibre of glass, with a diameter of $1 \mu \mathrm{m}$, acting as a torsion wire. The restoring torque corresponds to $2 \cdot 10^{-10} \mathrm{Nm}$. The torsion oscillation time is about six minutes. The torsion balance is installed in a glass container that can be evacuated down to $1 \mathrm{~Pa}$. To shield the balance from thermal radiation and electrical fields, it was surrounded by some sheath of metallized plastic foil.

A weak light source - vertically positioned and small - was observed via a telescope with a focus of $90 \mathrm{~cm}$ from a distance of $2.5 \mathrm{~m}$ (Fig.3). The movements of the pendulum are observed by a video camera which is fixed at the end of the telescope and the result is plotted by means of an x, t-recorder. By electronic methods a linear electrical signal in reference to the position of the incident light-beam was obtained. The signal is smoothed out by means of an active three-step Tschebytscheff filter, with integration times between 0.5 to $4 \mathrm{~s}$. To suppress signal-shifts, generated by slow superpositioned movements of the balance, the signal was finally differentiated by an R-C-component, to obtain the velocity and the direction of the rotation of the pendulum.

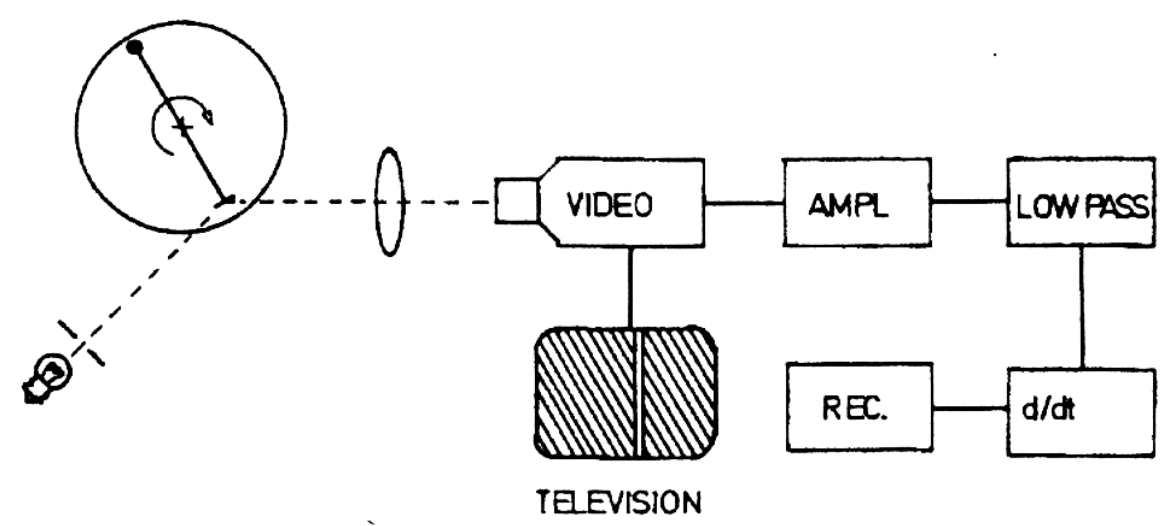

Fig.3. Scheme of the torsion balance measure system

Fig. 4 shows a test of the sensitivity of the torsion balance. The mutual gravitational attraction between a mass of $120 \mathrm{~g}$ and the masses of the torsion pendulum can be observed easily. The mass was positioned tangential in a distance of $4 \mathrm{~cm}$ to one mass of the pendulum and then taken away. About $100 \mathrm{~s}$ later the position of the mirror runs out of the detectable area of the measure system described above. The calculated acceleration of the pendulum masses is about $2.10^{-9} \mathrm{~m} / \mathrm{s}^{2}$. The ratio between noise and signal in Fig.4 indicates a resolution limit in the 
range of $10^{-11} \mathrm{~m} / \mathrm{s}^{2}$, which corresponds to the gravitational force of a mass of $\sim 1 \mathrm{~g}$ in the position described above. Hence, the torsion balance has the potential to detect local DTQ powerdensities even if the cross-section is much smaller than $10^{-5} \mathrm{~b}$.

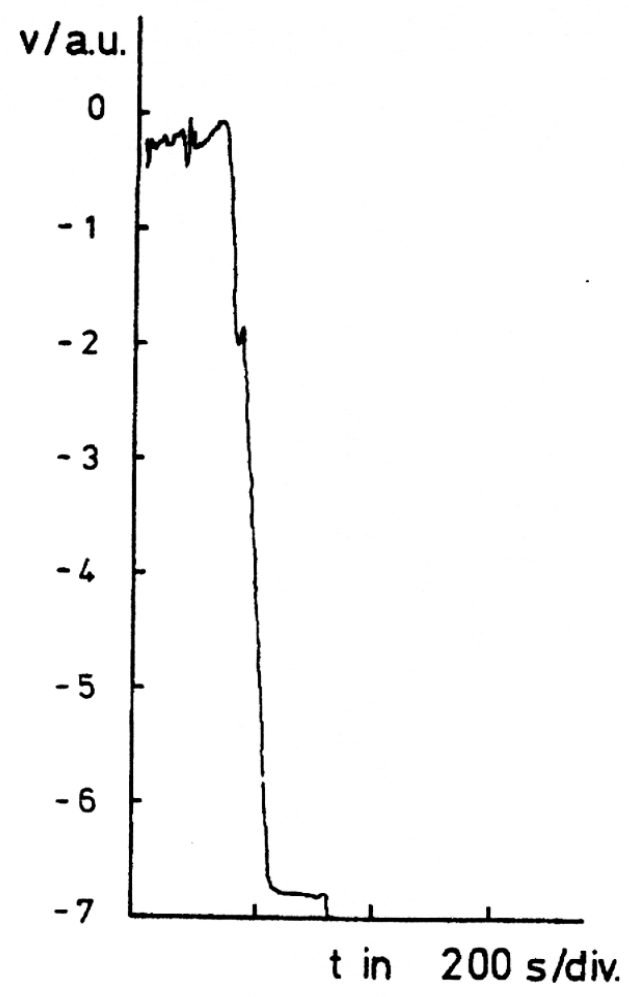

Fig.4 Sensitivity test of the torsion balance measuring system. A mass of $120 \mathrm{~g}$, positioned near to one mass of the pendulum, was taken away. The fluctuation of the signal corresponds to accelerations in the order of $10^{-11} \mathrm{~m} / \mathrm{s}^{2}$.

\subsection{The proof of DTQs in the experiment}

An optimal system to generate DTQ related forces would be a device similar to the one presented in the Mirror Mass Model in Chap.1 (see Fig.1). However, light forces of a single photon or even an intense light beam are much too weak to be of experimental use. So, one has to take, for instance, a mechanical oscillator and hope, that the DTQ flow in energy conservation processes is universal as already mentioned in the remarks at the end of Chap.3. Since the expected forces are very weak and difficult to distinguish from small movements of a torsion pendulum, a periodical experiment was preferred. A DTQ-source with alternating tangential flow direction has the potential to prove effects even in noisy surroundings as long as a periodic structure of the signal can be stated.

\subsubsection{Specification of the mechanical oscillator}

Experimental setup of the chosen mechanical oscillator is a flywheel of aluminium with a diameter of $48 \mathrm{~cm}$, a thickness of $2 \mathrm{~cm}$ and a weight of $10 \mathrm{~kg}$. The momentum of inertia is $0.28 \mathrm{~kg} \mathrm{~m}^{2}$. The drive of the flywheel is a cord of rubber fibres, $60 \mathrm{~cm}$ long and $3 \mathrm{~cm}$ in diameter. A sketch of the experimental setup is shown in Fig.5. The rubber drive was chosen to avoid diamagnetic effects in the torsion balance caused by magnetic fields and radiometer forces, caused by the thermal radiation of an electrical motor etc.. The torsion oscillation fre- 
quency of the flywheel amounts to $0.067 \mathrm{~Hz}$. The disk of the flywheel was positioned in the same plane as the torsion pendulum (see Fig.5). The distance between the disk and the masses of the pendulum varied between 1 and $4 \mathrm{~cm}$.

\subsubsection{Measurements}

Before each measurement, the disk of the flywheel had been rotated completely 10 times and fixed in this position until the torsion balance was in rest. In each case the torsion balance was filled with air. The gas pressure varies between 500 and $1013 \mathrm{hPa}$ mainly to suppress radiometer forces. However, the force under study affects not only the mass of the pendulum but also the mass of the gas-content in the glass vessel. A complex movement or rotation of the gas content in the vessel remains after each measurement (see below) and lasts for hours.

The stored energy can be estimated to $100 \mathrm{~J}$, and the average power to $10 \mathrm{~W}$.

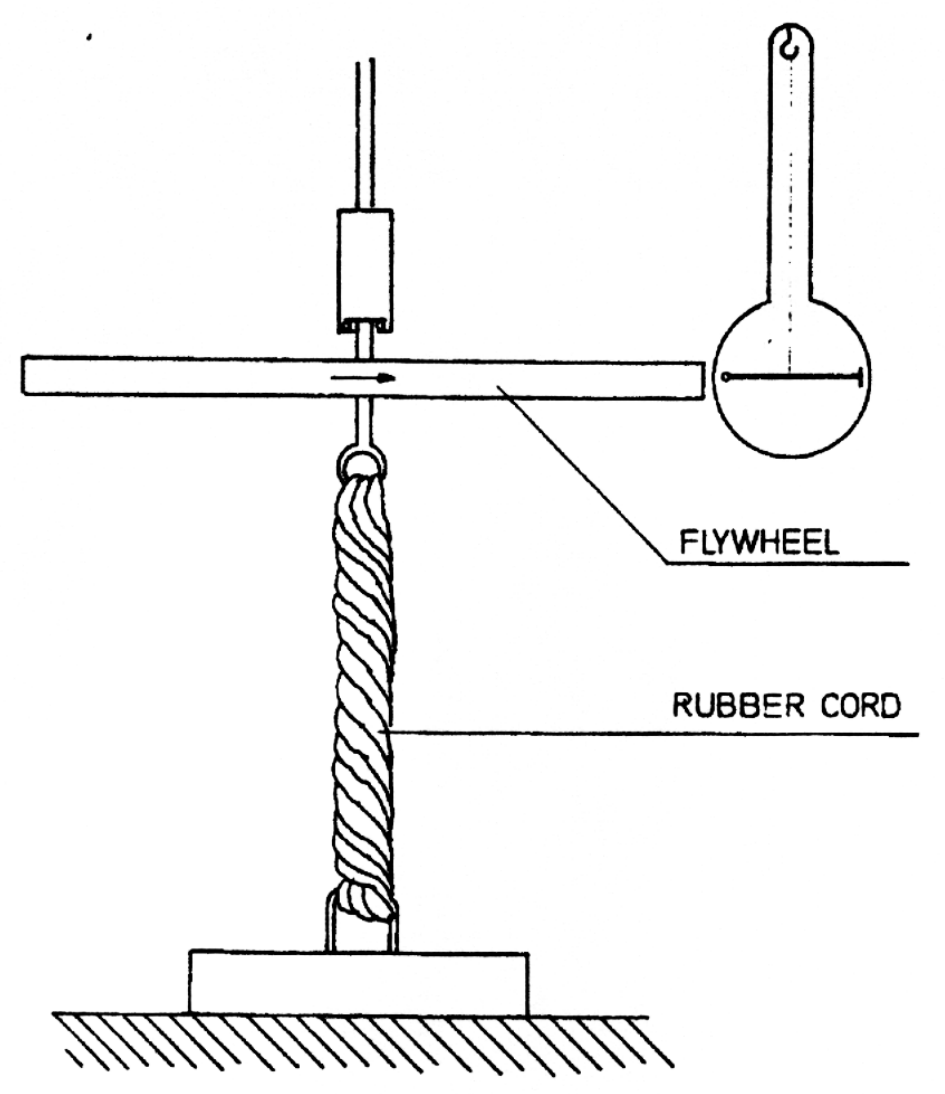

Fig.5 Side-view of the system made up of the flywheel and the torsion balance. The sense of rotation drawn in indicates the initial movement of the flywheel, which is opposite to the final movement of the pendulum.

In correlation with the beginning of the angular acceleration of the flywheel, relatively strong periodical signals were measured if the distance between the disk and the pendulum is in the order of $1 \mathrm{~cm}$. Figure 6 shows a plot of the signals recorded by an x,t-recorder. Although noisy in signal the periodicity and correlation to the flywheel parameter is convincing After each experiment the torsion pendulum rotated slowly $(1 \mathrm{~mm} / 60 \mathrm{~s})$ in the opposite direction to that of the flywheel in the beginning of the experiment (see Fig.6). This is interpreted as the global movement of the residual gas content in the vessel as a consequence of the forces acting on gas and pendulum. 
More distant positions between the flywheel and the pendulum mass show a roughly quadratic decreasing effect. Besides the metalized plastic foil which covered the glass vessel in some experiments, a $1 \mathrm{~mm}$ sheet of lead was fixed between the disk and the glass vessel. Statistically no reduction of the effect was observed.

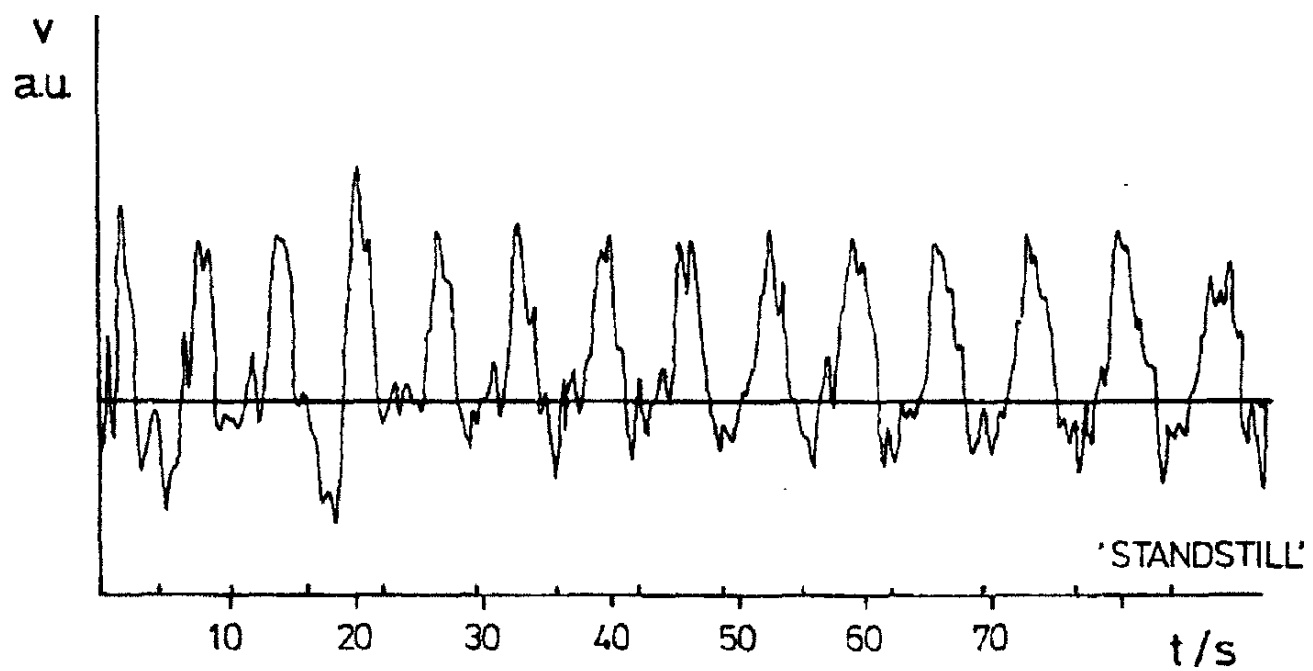

Fig.6. Periodical movement pattern of the torsion balance induced by tangential forces generated by the oscillating flywheel. The markings at the horizontal time axis indicate the measured standstill of the flywheel $(\omega=0)$. This even gives information about the sign of the DTQ radiation. The resulting force effect is repulsive.

\subsubsection{Discussion of the measured signals}

Figure 7 shows in principle - vertically arranged - the velocity of the pendulum mirror (here approximated by a $\sin (\mathrm{wt})$-function), the sign and intensity of the acting force on the lowdistant pendulum mass (mirror), the angular velocity $\omega$ and the angular acceleration $\dot{\omega}$ over a whole oscillation period of the flywheel. 


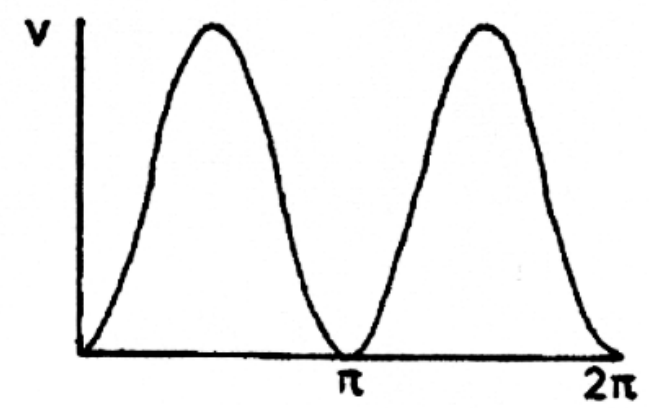

$F$

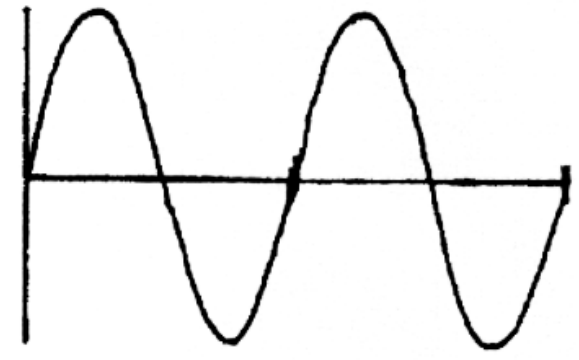

w

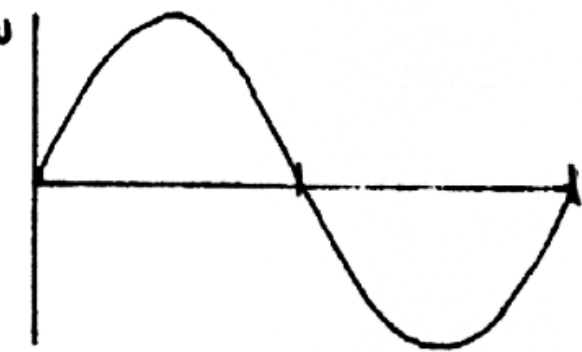

$\dot{\omega}$

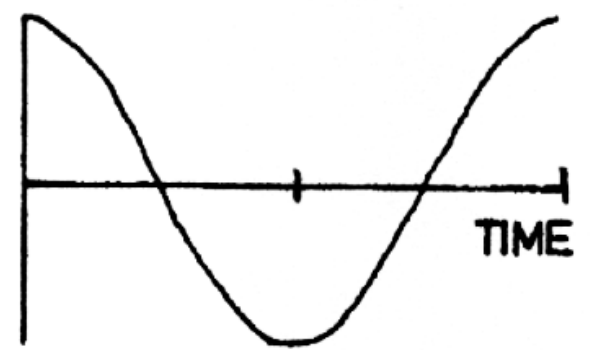

Induced velocity of pendulum $\square \omega \cdot \dot{\omega}$

Repelling- and attractive forces acting on the pendulum

$\omega$ of flywheel

$\square \omega \cdot \dot{\omega}$

Fig.7 With respect to a full oscillation period of the flywheel the measured velocity of the pendulum-mirror, acting attractive and repelling forces, angular velocity and acceleration are vertically arranged. The $\mathrm{x}$-axis is the time axis. A force proportional to $\omega \cdot \dot{\omega}$ seems to be responsible for this behaviour.

The force $\mathrm{F}$ can be interpreted as:

$$
F \propto \omega \cdot \dot{\omega}
$$

depending not only on the angular acceleration a the mass particles experiences in the flywheel $(\square \dot{\omega})$, but also on the actual angular velocity ( $\square \omega)$ of the mass particles. A reasonable ansatz for the force seems to be:

$$
F=\text { const } \cdot f(r) \cdot a \cdot v
$$


f (r): Distance law (see 4.2.2)

a : Average acceleration of the mass points in the flywheel
$\mathrm{v}:$ Average velocity of the mass points of the flywheel

Equation (20) follows from equation (17) if $\mathrm{j}$ is substituted:

$$
\mathrm{F}=\mathrm{F}_{0} \frac{\sigma}{\mathrm{A}} \cdot \frac{\mathrm{v}}{\mathrm{c}}
$$

Here $\sigma / \mathrm{A}$ represents the distance law and $\mathrm{v} / \mathrm{c}$ is the relativistic factor.

The average tangential force, that is to say the average tangential acceleration of a mass particle in the outer radius of the flywheel, can be estimated to $5 \mathrm{~m} / \mathrm{s}^{2}$, the average velocity to 2 $\mathrm{m} / \mathrm{s}$. Equation (20) yields an acceleration - corresponding to $\mathrm{F}$ - in the order of $3 * 10^{-8} \mathrm{~m} / \mathrm{s}^{2}$ .The measured acceleration is higher. With an estimated maximum velocity of the pendulum masses of $10^{-5} \mathrm{~m} / \mathrm{s}$, the accelerations must be in the order of $10^{-6} \mathrm{~m} / \mathrm{s}^{2}$.

In the vein of the assumptions above, the measured repelling acceleration hints to a crosssection in the order of $1 b$.

\section{Conclusion}

In conclusion, it is conjectured that:

- periodically accelerating and decelerating force effects have become visible in these experiments, i.e.,

- the DTQs emitted in the frame of energy conservation can be responsible for the force,

- the DTQs seem to establish an energy flow into or from space during energy conservation processes, which are measurable by means of interacting forces,

- the force does not only affect the pendulum weight but also the gas content in the vessel, which leads to an imprinted gas flow after the end of experiment,

- the negative result of the lead-shielding experiment (see Chap.4.2.2) hints to an 'Open Force' situation, where forces are carried out of the area of the interaction experiment

- the measurements are based on the periodicity of the DTQ-source in the flywheel process, which gives first reliable information about the chronology and the strength of the Doppler force.

In Ref. [10] an interesting experiment is reported which is based on a superconducting flywheel, which shows relatively strong effects in nearby located laser gyroscopes. These signals could be registered only during the phase of angular acceleration or deceleration. The interpretation as a gravitomagnetic effect seems many order too high as it could be in accordance with the Lense-Thirring effect (a factor of $10^{23}$ discrepancy to be a gravitomagnetic effect) [11]. Since the gyroscope measure only effects during acceleration and deceleration phases of the flywheel, it seems that DTQs can be responsible for this effect.

\section{Outlook}


A final remark concerns the quality of the DTQ pool. Since even extreme high energy conservation processes will take place in nuclear reactions as well as in collision processes in high energetic particle interactions, the pool seems to be filled even with highest DTQ energies isotropically and of course with high density. This is reminiscent of the zero-point radiation of electromagnetic waves in QED as was described for the first time by H.G. Casimir in 1940 [12]. Note the energy content of the zero-point radiation is unlimited provided that there is no cut-off frequency assumed as, for example, resulting from the Plank length. Note that DTQs are different to electromagnetic waves as for instant they are not screened but penetrate a sheet of lead. In addition to the zero-point radiation it seems that we have to deal at any position of the space with an isotropic distribution of DTQ energies which penetrates a probe.

In future works the experiments have to be repeated and the sensitivity of the detection system has to be improved. Periodic experiments should be preferred since interpretation is optimally reliable. The lack of calibration and the unconcernedly of direction and strength of force should then be overwhelmed in next experiments.

Acknowledgement

I would like to express my gratitude to V. Schultheiß, who made valuable contributions during the final reading of the draft.

\section{References}

[1] Mößbauer, R. Gammastrahlung in Ir191. In: Zeitschrift für Physik 151, 124-143 (1958), doi: $10.1007 / \mathrm{BF} 01344210$.

[2] Schrödinger, E. Dopplerprinzip und Bohrsche Frequenzbedingung, Phys. Z. 23, 301 (1922)

[3] Cantor W. Equivalence of Compton Effect and Doppler Effect, Stroboscopic Letters 4 (3 \& 4), 59 (1971)

[4] Kidd R., Ardini J., Anton A., Compton effect as a double Doppler shift, Am. J. Phys. 53 (7), 641 (1985)

[5] Lemons D. S., Doppler shift and stellar aberration from conservation laws applied to Compton shift, Am. J. Phys. 59 (11), (1991)

[6] Wilkins D., A new angle on Compton scattering, Am. J. Phys. 60 (3), 221 (1992)

[7] Ashwood D. JG., Jennison JG R: C:, The scattering of an electromagnetic wave by a free electron, J . Phys. A: Math. Nucl. Gen.7, No. 7, 803 (1994)

[8] Schultheiss C., Mass Attraction Caused by Ultralong-Wave Photons?, Adv. Studies Theor. Phys., 2, no. 10, 491-505 (2008)

[9] Schultheiss C., Momentum and Energy of a Mass Consisting of a Confined Photon and Resulting Quantum-Mechanical Implications, Adv. Studies Theor. Phys. 7 no.9-12, 555$583(2013)$

[10] Tajmar M., Plesescu F., Seifert B., \& Marhold K. AIP Conference Proceedings 880, 1071 (2007); https://doi.org/10.1063/1.2437552 
[11] Graham, R.D.,Hurst R.B.,Thirkettle R.J, Rowe C.H,.Butler P.H., Experiment to detect frame dragging in a lead superconductor, Elsevier 468, 5, 1, 383-387 (2008)

[12] Casimir H.B.G., Koinkl. Ned. Akyd. Wetens Sect. Proc. 51, 793 (1948) 
Figures

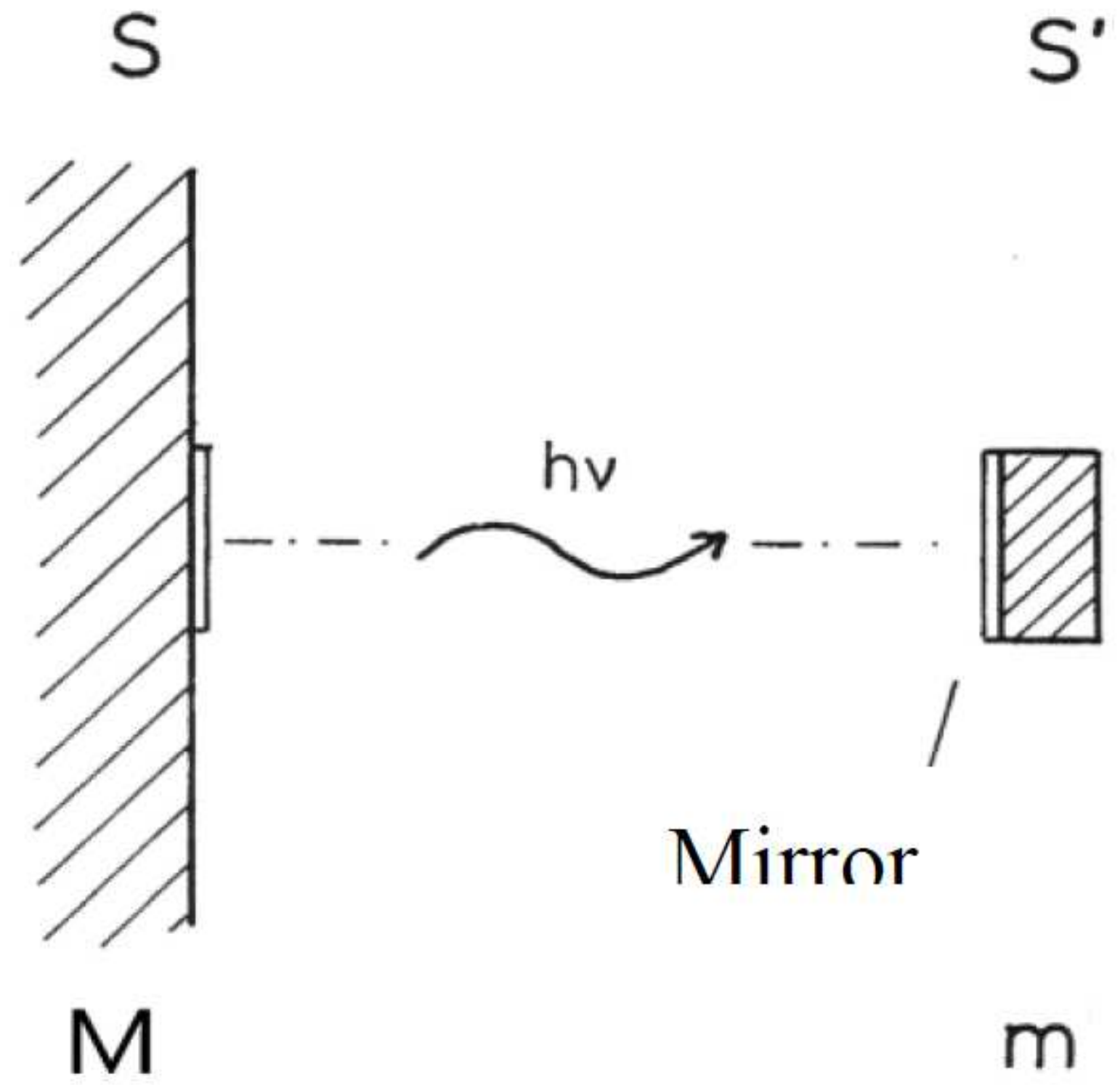

Figure 1

Arrangement of mirrors, masses and photon in the Mirror Mass Model 

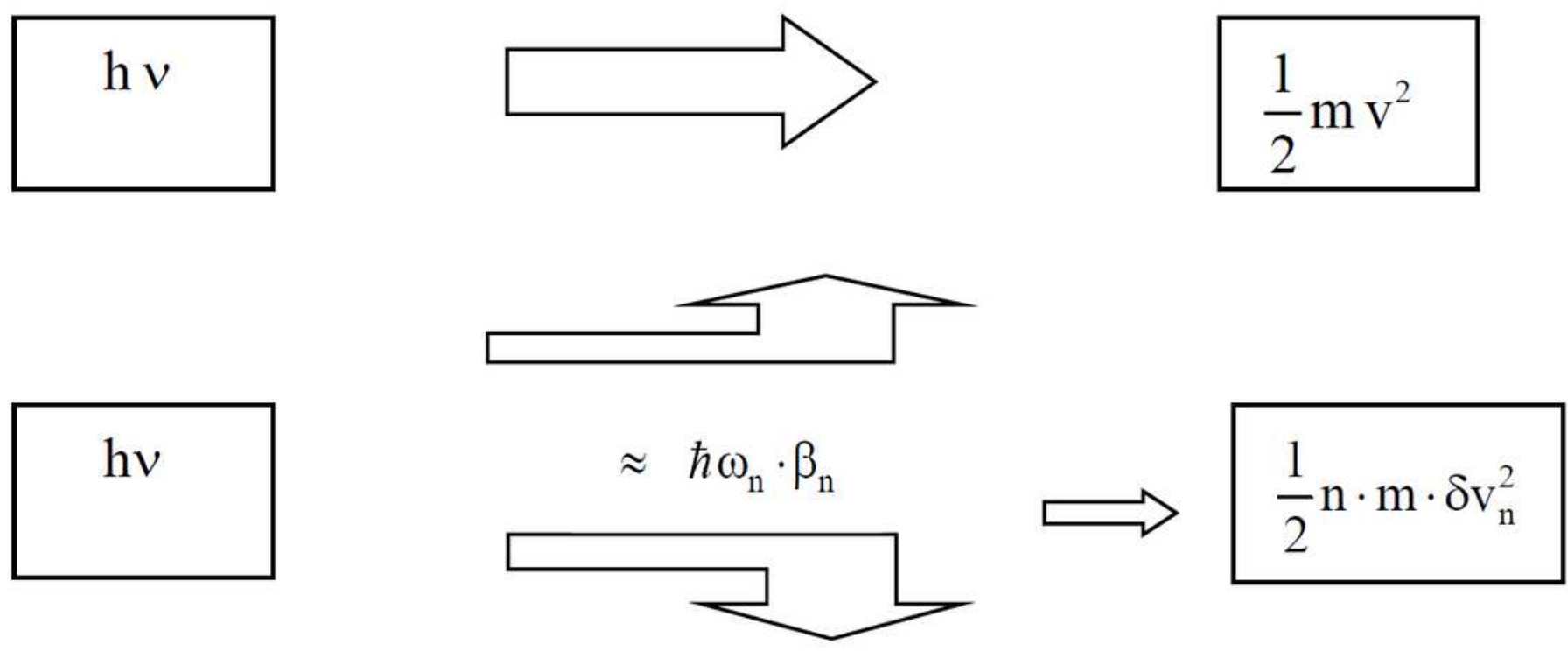

\section{Figure 2}

Classical scheme of energy transfer of a photon to a mass $m$ during a photoelectric effect (upper graph), while in the microscopic model the flow of energy into the space is dominant in comparison with the direct step-wise flow on $\mathrm{m}$. This is true especially in non-relativistic processes (see Eq.14). The mass $\mathrm{m}$ itself gains a velocity push ( $2 \delta \mathrm{vn})$ with each collision and approaches successively the final velocity $\mathrm{v}$ after $\mathrm{n}$ reflections.

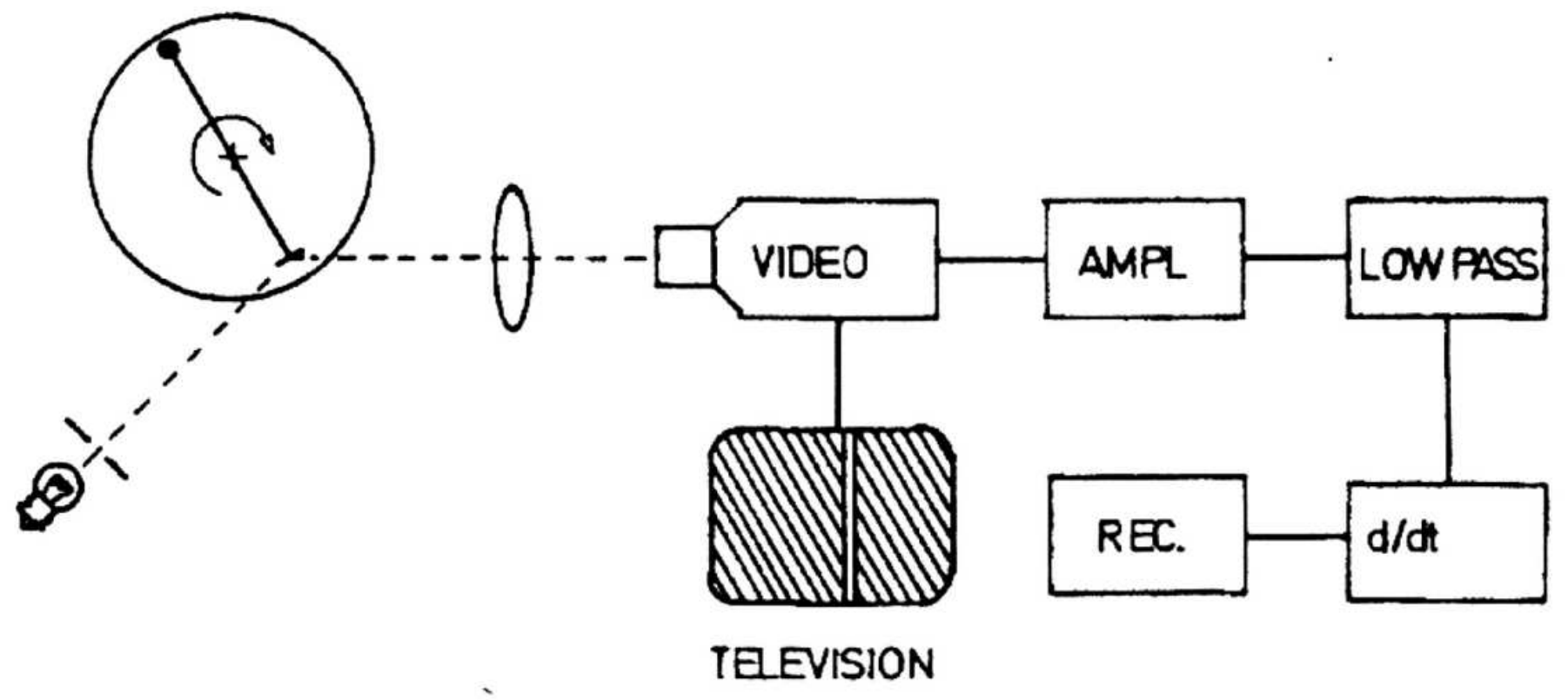

Figure 3 
Scheme of the torsion balance measure system

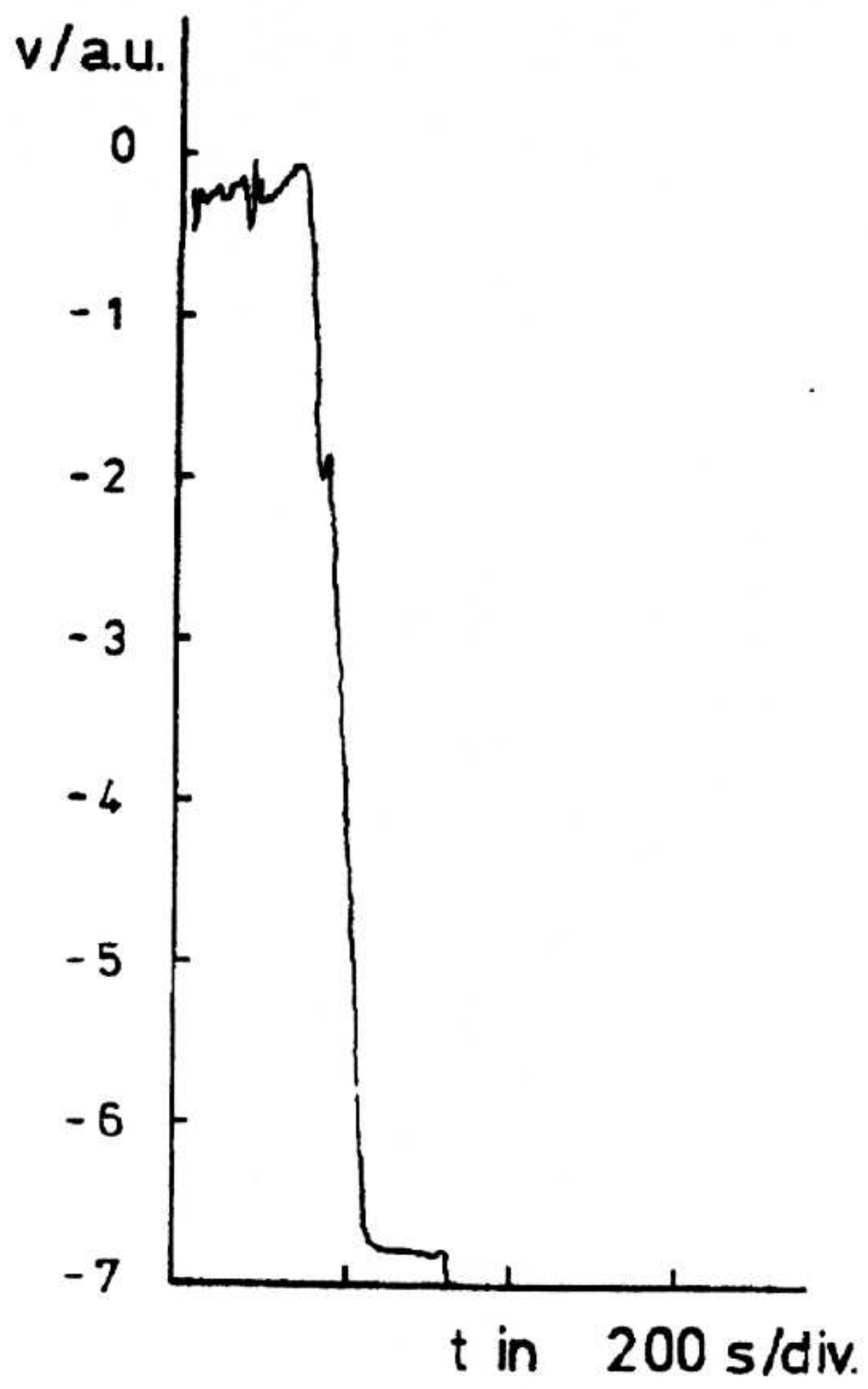

Figure 4

Sensitivity test of the torsion balance measuring system. A mass of $120 \mathrm{~g}$, positioned near to one mass of the pendulum, was taken away. The fluctuation of the signal corresponds to accelerations in the order of $10-11 \mathrm{~m} / \mathrm{s} 2$ 


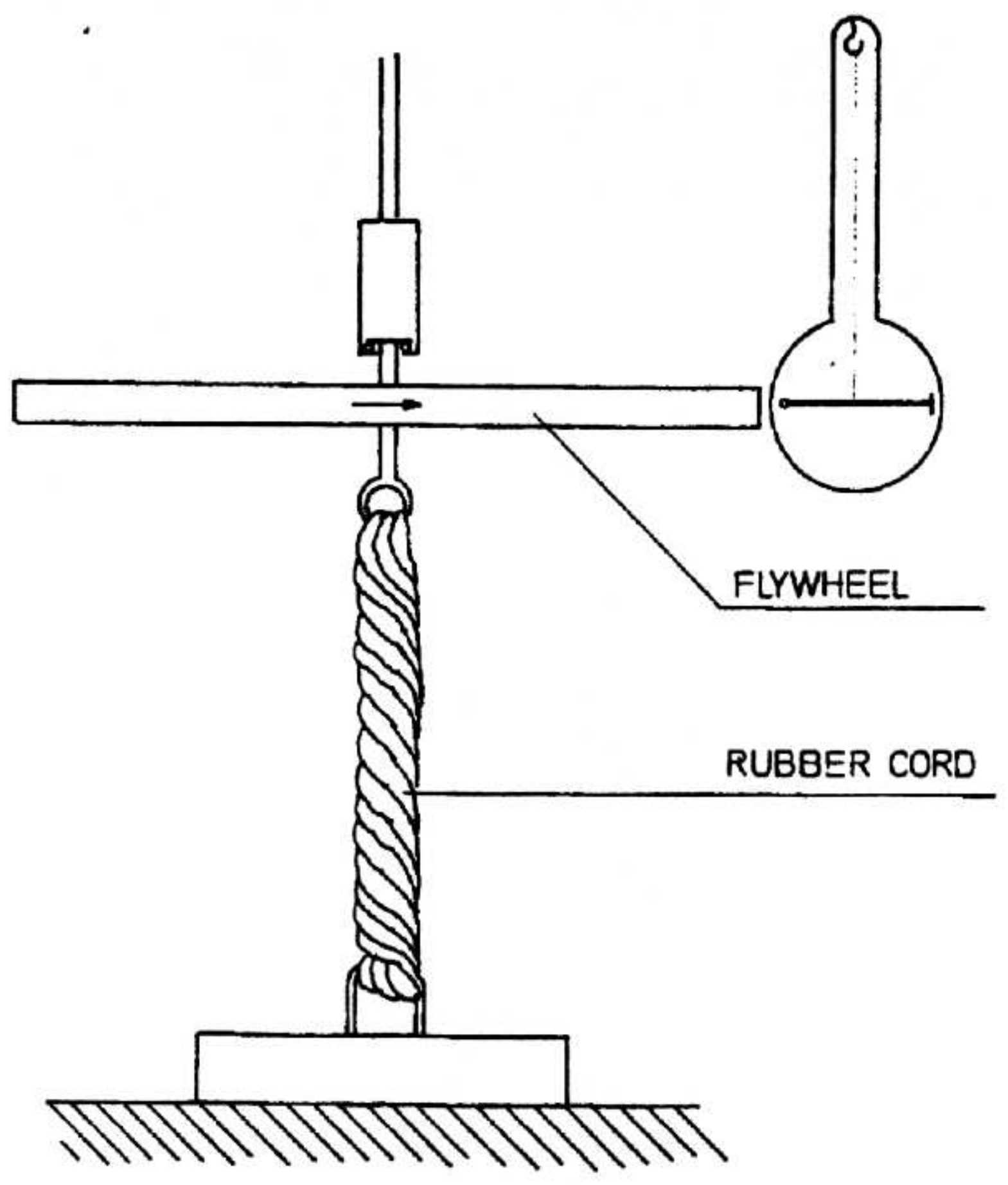

Figure 5

Side-view of the system made up of the flywheel and the torsion balance. The sense of rotation drawn in indicates the initial movement of the flywheel, which is opposite to the final movement of the pendulum. 


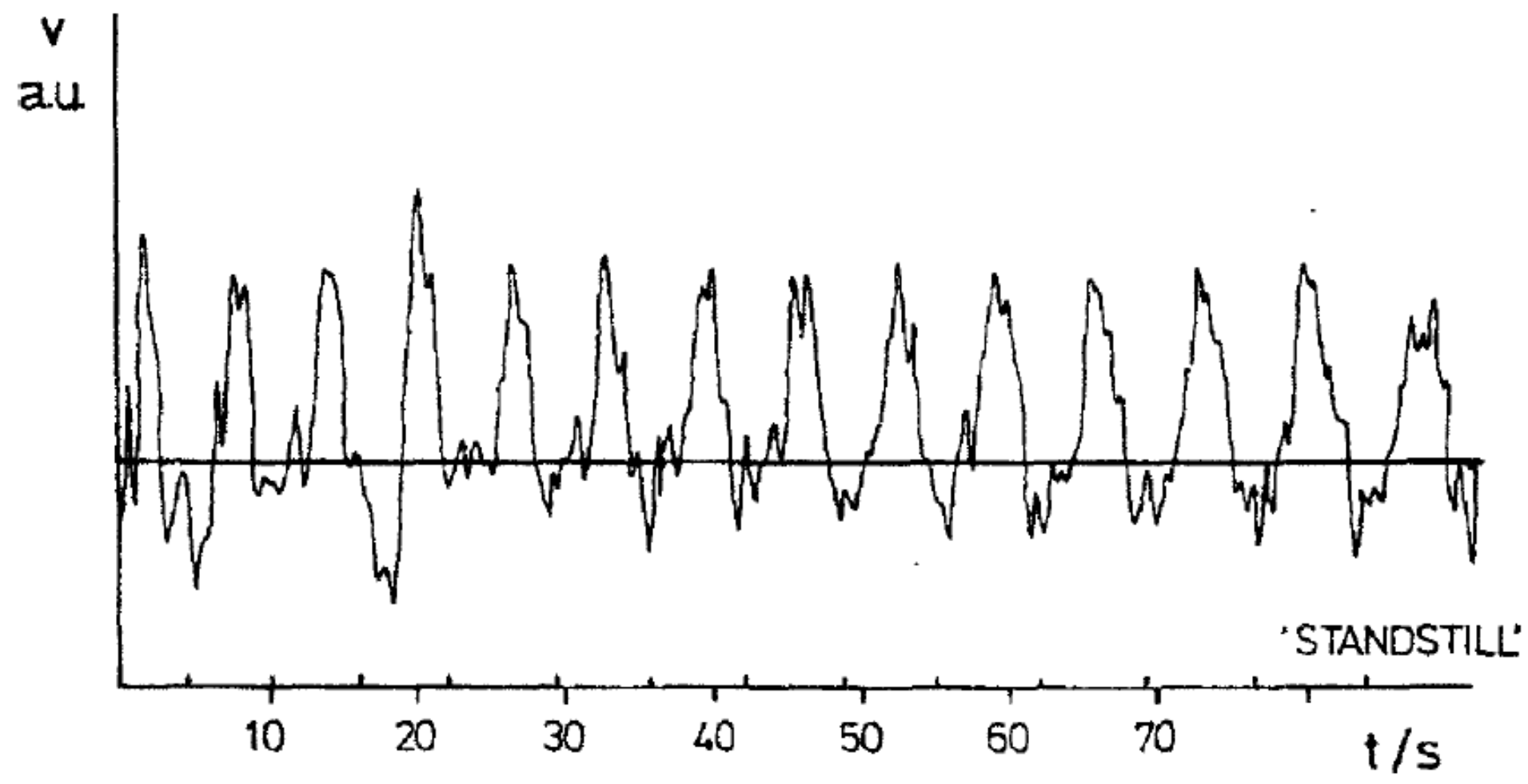

Figure 6

Periodical movement pattern of the torsion balance induced by tangential forces generated by the oscillating flywheel. The markings at the horizontal time axis indicate the measured standstill of the flywheel $(w=0)$. This even gives information about the sign of the DTQ radiation. The resulting force effect is repulsive. 


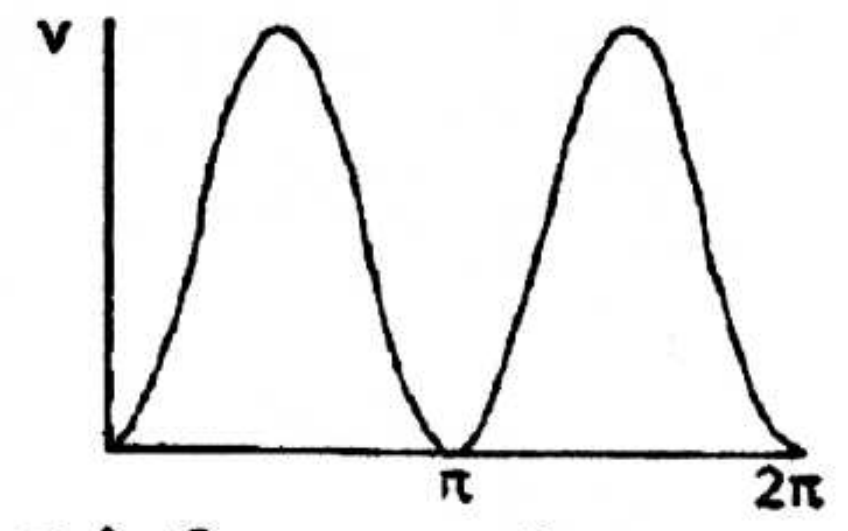

\section{Induced velocity of pendulum $\square \omega \cdot \dot{\omega}$}

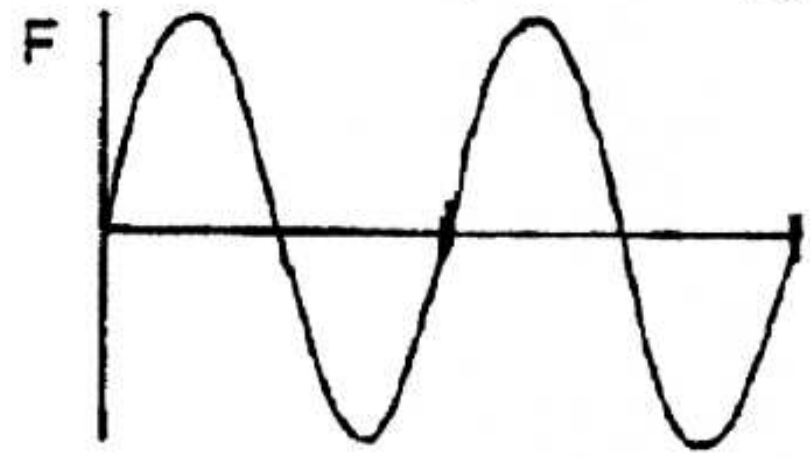

Repelling- and attractive forces acting on the pendulum

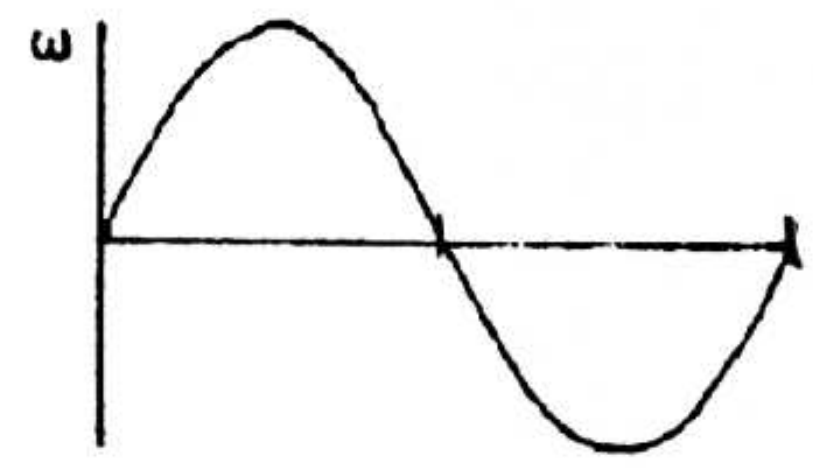

$\omega$ of flywheel

$\square \omega \cdot \dot{\omega}$

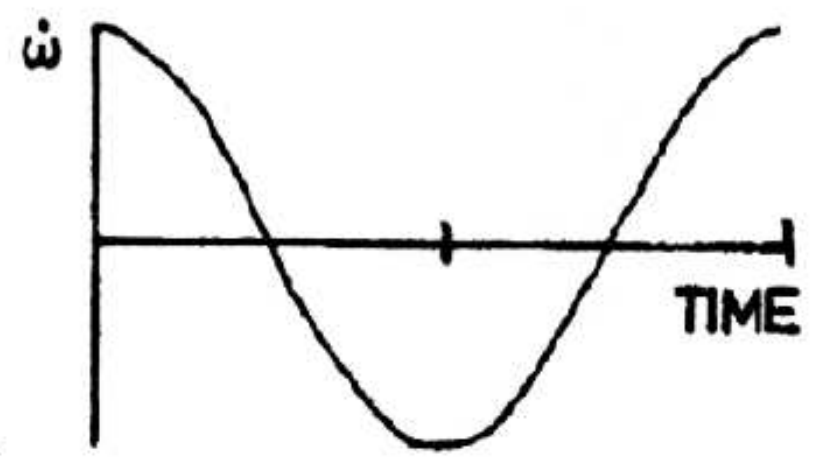

$\dot{\omega}$ of flywheel

\section{Figure 7}

With respect to a full oscillation period of the flywheel the measured velocity of the pendulum-mirror, acting attractive and repelling forces, angular velocity and acceleration are vertically arranged. The $x$-axis is the time axis. A force proportional to $\mathrm{w}-\mathrm{w}$ seems to be responsible for this behaviour. 